\title{
Expression of COX - 2 in Oral Leukoplakia Using RT-PCR: A Molecular Study
}

\author{
N Rakesh, ${ }^{1}$ Asha R Iyengar, ${ }^{2} \mathrm{~K}$ S Nagesh, ${ }^{3}$ Deepa B Patil, ${ }^{4}$ Vidya G S ${ }^{5}$ \\ ${ }^{I}$ PhD Research Scholar Dept. Oral Medicine,Diagnosis \& Radiology DAPM R V Dental College, Bangalore. \\ ${ }^{2}$ Professor \& HOD Dept. Oral Medicine,Diagnosis \& Radiology DAPM R V Dental College, Bangalore. \\ ${ }^{3}$ Director Dept. Public Health RGUHS, Bangalore. \\ ${ }^{4}$ Postgraduate Student Dept. Oral Medicine,Diagnosis \& Radiology M S Ramaiah Dental College \& Hospital, \\ Bangalore. \\ ${ }^{5}$ M. D. S Sree NRJ Specialist's Dental Clinic, Bangalore.
}

\begin{abstract}
Background: Oral leukoplakia is a potentially malignant disorder that in a small but significant proportion of cases changes into oral cancer. Literature review suggests the association of inflammatory mediator such as cyclooxygenase-2(COX-2) with oral cancer. Expression of COX-2 gene in the oral potentially malignant disorders and oral cancer may add on a light to the existing diagnostics and therapeutics of these oro-mucosal diseases.

Aim: To compare the expression of COX-2 gene in oral leukoplakia with that of controls.

Methodology: The present study comprised of 40 subjects. 20 subjects each in oral leukoplakia \& controls. Tissue samples were collected at the time of biopsy for the clinically diagnosed cases of leukoplakia and also from control subjects and stored in RNAase free containers. RNA was isolated and COX-2 gene expression was noted through RT-PCR method.
\end{abstract}

Results: Demographic details were expressed in mean \pm standard deviation. Mann-Whitney test was applied to correlate the COX-2 expression between the groups and other parameters. A statistically significant higher expression of COX-2 was found in oral leukoplakia $(0.739 \pm 0.078)$ when compared to controls $(0.138 \pm 0.103)$ with $P<0.000$.

Conclusion: COX-2 expression can be used as an adjunct to assess the severity of oral leukoplakia \& for early diagnosis of malignant diseases.

Keywords: Oral leukoplakia, Inflammation, COX-2, RT-PCR, Malignant potential

\section{Introduction}

Oral Leukoplakia is a clinical term used when any other white oral lesion has been excluded. It is defined as "a predominantly white lesion of the oral mucosa that cannot be characterised as any other definable lesion". Tobacco smoking is thought to be a responsible etiological factor, but idiopathic forms of oral leukoplakia also do exist. ${ }^{1}$ The role of alcohol, viruses and systemic conditions need further investigations. ${ }^{2,3}$ Oral leukoplakia is not uncommon. Even though it varies significantly among geographical areas and demographical groups, the prevalence of it in the general population usually varies from less than $1 \%$ to more than $5 \% .{ }^{4}$

The rate of malignant transformation of oral leukoplakia into oral cancer varies from almost $0 \%$ to about $20 \%$ in 1 to 30 years. ${ }^{4}$ Recently, a study investigating the natural limit of oral leukoplakia malignant transformation on the basis of European epidemiological data, concluded that the upper limit of the annual transformation rate of oral leukoplakia is unlikely to exceed $1 \% .{ }^{5}$ Non-homogeneous oral leukoplakias carry a higher degree of risk of transformation when compared with the homogeneous variants.

Since most oral leukoplakias are asymptomatic, the need for treatment is primarily based on the precancerous nature of the lesion. Prevention of malignant transformation is particularly important in view of the poor prognosis associated with oral cancer, with only $30 \%$ to $40 \%$ of patients still alive 5 years after the diagnosis. ${ }^{6}$ Clinical, histological and molecular markers may contribute in assessing the risk of an individual to develop cancer, however a single, evidence-based and clinically useful predictor of malignant transformation for dysplastic and non-dysplastic leukoplakias, is not available at the moment.

Cyclooxygenases (COX's) are the rate limiting enzymes for high output production of prostanoids (Prostaglandins and Thromboxanes ) from arachidonic acid which play an important role in various pathophysiological conditions. Enhanced synthesis of Prostaglandins, which results from upregulation of COX2 , increases the proliferative activity of neoplastic cells, cancer invasiveness and metastasis, promotes angiogenesis and inhibits immune surveillance and apoptosis. 
In recent years, over-expression of COX-2 has been reported in various cancers from colon, stomach, breast, esophagus, pancreas and oral cancer. ${ }^{7-11}$ They play an important role in initiation and progression of carcinomas of various organs. Although many studies have shown over-expression of COX -2 in oral malignant lesions, only few researchers have compared COX-2 in oral potentially malignant disorders \& normal mucosa.

An improved understanding of molecular mechanism of disease initiation and progression of oro mucosal diseases would also help to delineate further therapeutic modalities or chemoprevention. The analysis of DNA, RNA, and proteins, obtained from diagnostic specimens, is currently revolutionizing the practice of surgical pathology and heralds a new era of diagnostic and prognostic tests that will greatly influence our dayto-day clinical decision making. The diagnosis of oro-mucosal diseases is fundamentally based on the microscopic study of cells and tissues. This diagnostic method remains the standard by which all other diagnostic tests are measured.

Today, PCR is the single technique that is used almost universally to permit the study of DNA and RNA obtained from a variety of tissue sources. PCR helps in the study of oro-mucosal diseases and provide clearer understanding of their pathogenesis. Since oral leukoplakia has got higher malignant transformation rate, thus in the present study, we have made an attempt to compare the molecular expression profile of COX-2 in oral leukoplakia and normal oral mucosa using real time - polymerase chain reaction (RT- PCR) method.

\section{Materials And Methods:}

The study was carried out from 2009 - 2013 and was approved by ethics committee and institutional board. The present study comprised of 40 subjects with age range $20-35 y$ rs. The study subjects were categorized into two groups - Group 1: Subjects diagnosed with oral leukoplakia (20), Group 2: Normal healthy subjects (20).

Subjects clinically and histopathologically diagnosed with oral leukoplakia who have not undergone any sort of treatment for the same were included in group 1 whereas age and gender matched subjects with normal oral epithelia devoid of any deleterious oral habits were considered in group 2 . The present study has excluded the subjects with any other concomitant diseases or syndromes apart from oral leukoplakia and also, subjects who were under treatment or who have previously undergone or currently on treatment for oral leukoplakia were excluded from the study.

Informed consent was obtained from each subject. Complete medical history and habit history was recorded. Tissue specimens were collected from clinically diagnosed cases of leukoplakia during minor surgical procedure (Biopsy) and also from normal healthy individuals for evaluating the expression of COX - 2 gene. Tissues were collected and transported in RNAse - free plastic vials and RNA was extracted immediately in the laboratory. In the present study we have incorporated the procedure for RNA extraction, PCR, \& RT - PCR as suggested by Pandey et al. ${ }^{12}$ in their study.

\section{Statistical Analysis:}

Demographic details were expressed as mean \pm standard deviation. The significance of COX -2 expression between the two study groups was calculated using Mann- Whitney test. The Statistical software IBM, SPSS 20.0, was used for the analysis of the data. Microsoft word and excel have been used to generate graphs, tables etc.

\section{Results:}

A total of 40 subjects were studied who have been equally categorised into two groups as mentioned in the above section. The total mean age of the subjects studied was (27.93 \pm 3.050$)$ years. 10 males and 10 females were included in both the groups (Table 1). In oral leukoplakia mean COX -2 expression was found to be $(0.739 \pm 0.078)$ while it was $(0.138 \pm 0.103)$ in controls as given in table 2 , graph 1 . Table 3 gives the description of COX -2 expression according to age, gender and habits in both the study groups. Graphical representation of the same has been shown in graph 2,3,4.

\section{Discussion:}

Malignant neoplasms are major causes of fear, morbidity and mortality all over the world. Globally 'oral cancer' is the sixth most common cause of cancer-related death. Oral cancer accounts for approximately $30-40 \%$ of all cancers in India. ${ }^{13}$

Leukoplakia is the most common oral potentially malignant disorder. Etiopathogenesis of oral leukoplakia encompasses two broad categories namely, oral leukoplakia of unknown etiology or idiopathic and oral leukoplakia associated with tobacco use. ${ }^{14}$ It is more often found among older and elderly men, and its prevalence increases with age advancement. It has been estimated that less than $1 \%$ of the affected men are younger than 30 years old and that the prevalence increases to $8 \%$ in male patients older than 70 years old and to $2 \%$ in female patients of 70 years or more. Although rates of malignant transformation may vary among studies, 
probably due to differences in diagnostic criteria and follow up intervals, the morbidity and mortality associated with oral cancer make oral leukoplakia a serious health problem.

Oral leukoplakia has got high chances of turning into malignancy. Oral leukoplakia located on the floor of the mouth, soft palate, and tongue are considered as high-risk lesions, while, in other areas, they may be considered as of low malignancy risk. ${ }^{15}, 16$ Thus, epidemiological studies have indicated an early intervention strategy to achieve reduced oral cancer related deaths. The discovery of immunological markers at clinical, histological and molecular level has marked the end of an era of groping in the dark for clues to the basis of cancer. Significant reduction in mortality can be achieved by advances in early diagnosis and implementation of multidisciplinary treatment programmes leading to improvement of survivorship and better quality of life. ${ }^{17}$

Different studies ${ }^{12,18-22}$ have demonstrated overexpression of COX-2 in premalignant and malignant oral lesions related to the degree of epithelial dysplasia. However, few studies ${ }^{21,22}$ have analyzed the expression of COX-2 in OLP. In a study by Lysitsa et al., ${ }^{22}$ they did not recognize differences in the epithelial expression of COX-2 when comparing the different degrees of activity of distinct clinical types of OLP. Others ${ }^{21}$ also did not find differences in the epithelial expression of COX-2 in OLP samples with different evolution time and prognosis outcome, or when they were compared with dysplastic lesions and oral squamous cell carcinoma (OSCC).

A study by Pandey et.al have found overexpression of COX -2 in OPMDs \& OSCC but they stated that overexpression of COX -2 is independent of stage of differentiation of disease. ${ }^{12}$ In our study also we have got results suggesting overexpression of $\mathrm{COX}-2$ in oral leukoplakia. And we also have found statistically insignificant correlation among oral habits and $\mathrm{COX}-2$ expression.

\section{Figures \& Tables}

Table 1: Demographic details of study subjects

\begin{tabular}{|l|l|l|}
\hline & Oral leukoplakia & Controls \\
\hline Males & 10 & 10 \\
\hline Females & 10 & 10 \\
\hline Mean Age (in years) & $\mathbf{3 0 . 1 5} \pm 2.007$ & $\mathbf{2 5 . 7 \pm 2 . 1 5 5}$ \\
\hline Habits & & \\
\hline Smokeless tobacco & 11 & - \\
\hline Smoking tobacco & 9 & - \\
\hline
\end{tabular}

Table 2: Distribution of patients by COX 2 RT-PCR values

\begin{tabular}{|l|l|l|l|l|}
\hline & $\mathbf{N}$ & Mean expression & SD & P \\
\hline Oral leukoplakia & $\mathbf{2 0}$ & $\mathbf{0 . 7 3 9}$ & $\mathbf{0 . 0 7 8}$ & $\mathbf{0 . 0 0 0}^{*}$ \\
\hline Controls & $\mathbf{2 0}$ & $\mathbf{0 . 1 3 8}$ & $\mathbf{0 . 1 0 3}$ & \\
\hline
\end{tabular}

*Statistically significant

Table 3: Expression of COX -2 among various parameters

\begin{tabular}{|l|l|l|}
\hline Variables & COX -2 in Oral leukoplakia & COX -2 in controls \\
\hline Age & - & $0.148 \pm .135$ \\
\hline $20-25$ years & $0.743 \pm 0.097$ & $0.129 \pm 0.633$ \\
\hline 26- 30 years & $0.734 \pm 0.043$ & - \\
\hline S1 -35 years & $\mathbf{0 . 7 3 7 \pm 0 . 1 0 0}$ & $\mathbf{0 . 1 5 2} \pm .144$ \\
\hline Male & $\mathbf{0 . 7 4 2 \pm . 0 5 4}$ & $\mathbf{0 . 1 2 4} \pm .036$ \\
\hline Female & $\mathbf{0 . 7 3 7 \pm 0 . 0 5 4}$ & - \\
\hline Habits & - \\
\hline Smokeless tobacco & $0.742 \pm 0.105$ & \\
\hline Smoking tobacco &
\end{tabular}

Graph 1: Mean COX - 2 expression in two study groups

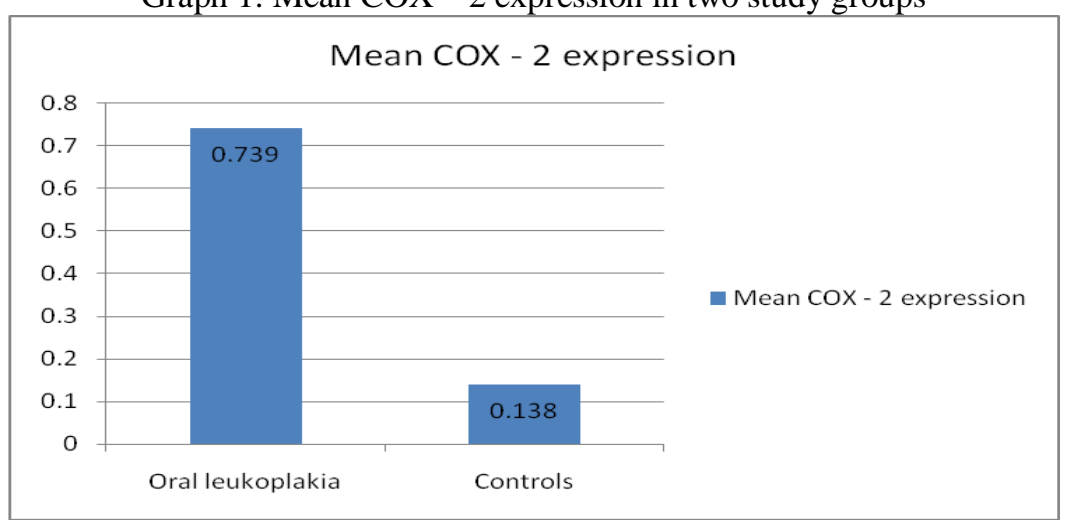


Graph 2: Comparison of $\mathrm{COX}-2$ expression in the study groups according to age.

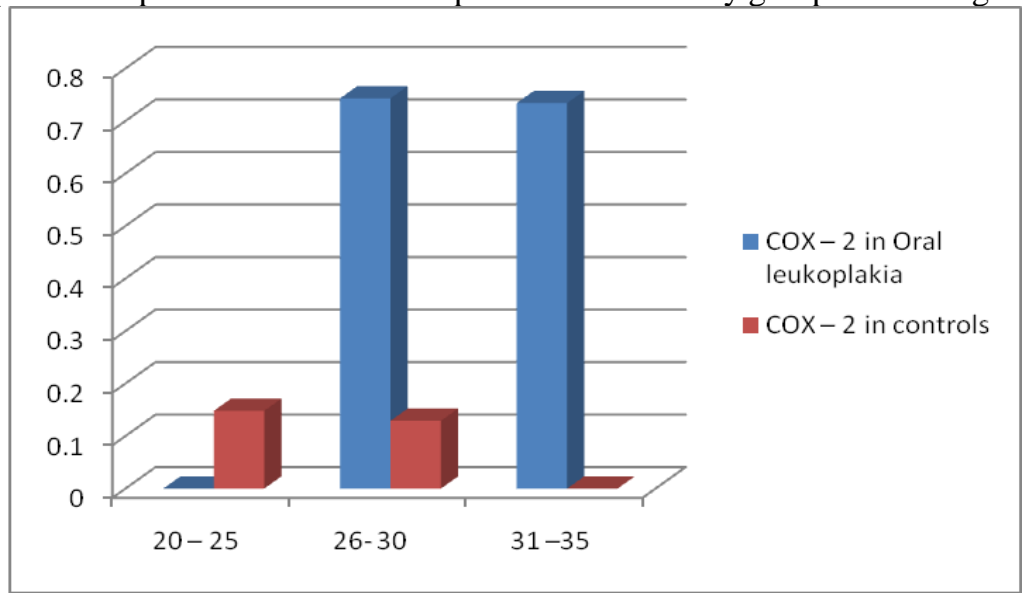

Graph 3: Comparison of COX -2 expression in the study groups according to gender.

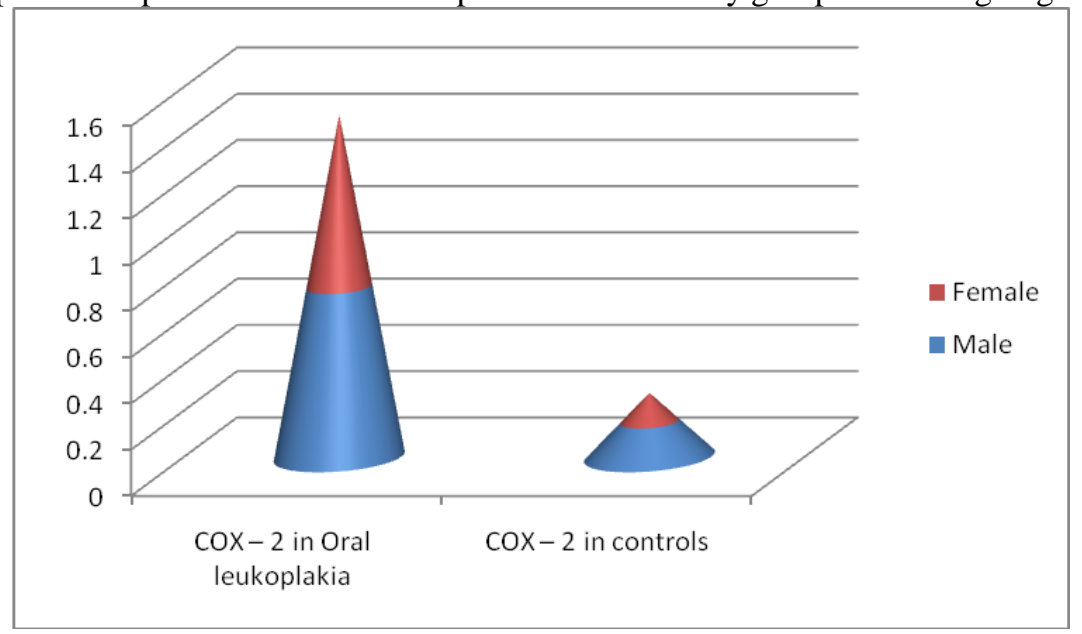

Graph 4: Comparison of COX -2 expression in the study groups according to habits.

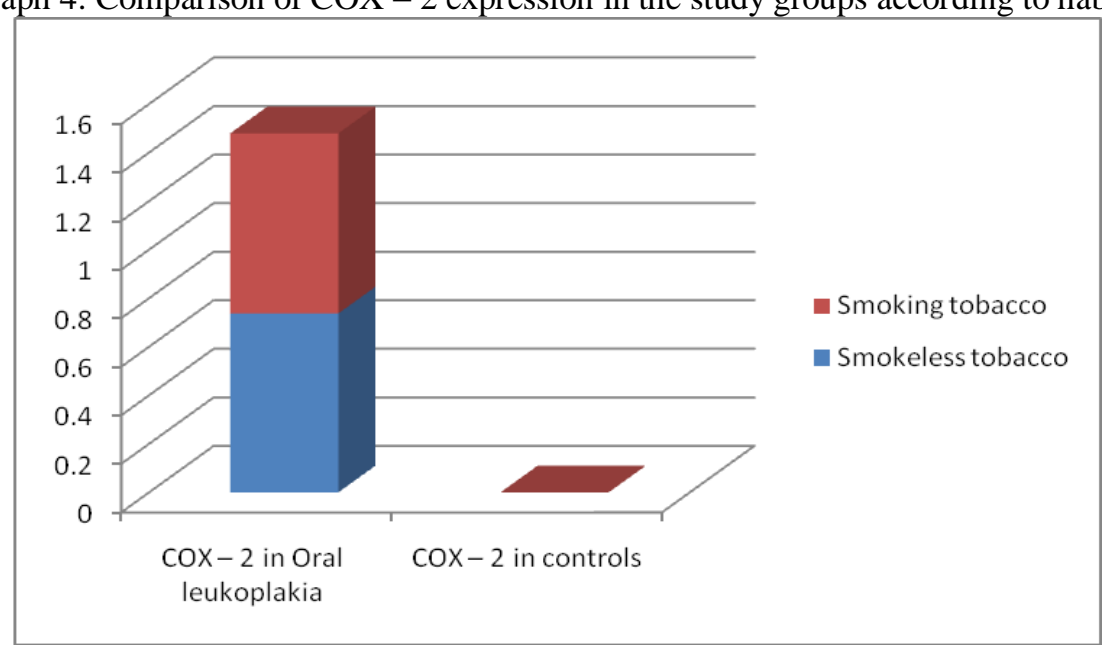

VII. Conclusion

Since most leukoplakias are asymptomatic, therefore oral diagnostician's vital role should be aimed to prevent such malignant transformation. Thus, we can conclude that COX-2 is overexpressed more frequently in oral leukoplakia. These results would be helpful in differentiating the cases so as to take useful therapeutic and preventive measures. Further longitudinal studies are needed to assess the long term follow-up of these patients to demonstrate the usefulness of the expression of COX-2 as a biological marker in oral leukoplakia. 


\section{References:}

[1]. Axell T., Occurrence of leukoplakia and some other oral white lesions among 20,333 adult Swedish people, Community Dentistry and Oral Epidemiology,15(1), 1987,46-51.

[2]. Campisi G, Giovannelli L, Arico P, Lama A, Di Liberto C, Ammatuna P, et al., HPV DNA in clinically different variants of oral leukoplakia and lichen planus, Oral Surgery, Oral Medicine, Oral Pathology, Oral Radiology, and Endodontics, 98(6), 2004, 705711.

[3]. Dietrich T, Reichart PA, Scheifele C., Clinical risk factors of oral leukoplakia in a representative sample of the US population, Oral Oncology, 40(2), 2004, 158-163.

[4]. Lodi G, Sardella A, Bez C, Demarosi F, Carrassi A., Interventions for treating oral leukoplakia (Review), The Cochrane Collaboration and published in The Cochrane Library,(4), 2008.

[5]. Scheifele C, Reichart PA. Is there a natural limit of the transformation rate of oral leukoplakia?. Oral Oncology, 39(5), 2003, 470475 .

[6]. Scully C, Porter S. ABC of oral health. Oral cancer. BMJ, 321 (7253), 2000, 97-100.

[7]. Liu JF,Zhang SW,Jamieson GG,Zhu GJ,Wu TC,Zhu TN et al.The effect of COX2 inhibitor meloxicam on squamous cell carcinoma of the esophagus in vivo.International Journal of Cancer,122(7), 2008, 1639-1644.

[8]. Kawata R,Hyo S,Araki M,Takenaka H.Expression of cyclooxygenase-2 and microsomal prostaglandin synthase-1in head and neck squamous cell carcinoma.Auris Nasus Larynx, 37(4), 2010, 482-487.

[9]. Tucker ON,Dannenberg AL,Yang EK,Zhang E,Teng L.DalyJM.et. al. Cyclooxygenase-2 expression is up-regulated in human pancreatic cancer.Cancer Res, 6, 2000, 2424-2430.

[10]. Ristimaki A,Honkanen N, Jankala H, Sipponen P, Harkonen M. Expression of cyclooxygenase-2 in human gastric carcinoma.Cancer Res, 57, 1997, 1276-1280.

[11]. Waddell,W.R. et al. Sulindac for polyposis of the colon.J Surg Oncol, 24, 1983, 83-87.

[12]. M Pandey, O. Prakash, W.S.Santhi, C.S.Soumithran, R.M.Pillai. Overexpression of COX- 2 gene in oral cancer is independent of stage of disease and degree of differentiation. Int. J. oral Maxillofac. Surg., 37, 2008, 379-383.

[13]. Charushila Y. Kadam, Raghavendra V. Katkam, Adinath N. Suryakar, Kashinath M. Kumbar, Dipali P. Kadam. Biochemical markers in oral cancer. Biomedical Research, 22 (1), 2011, 76-80.

[14]. Adriana Spinola Ribeiro, Patr'1cia Ribeiro Salles, Tarc'1lia Aparecida da Silva, and Ricardo AlvesMesquita. A Review of the Nonsurgical Treatment of Oral Leukoplakia. International Journal of Dentistry, 2010, 2010, 1-10.

[15]. B. W. Neville and T. A. Day. "Oral cancer and precancerous lesions," CA: A Cancer Journal for Clinicians, 52(4), 2002, $195-215$.

[16]. L. Zhang, K.J. Cheung Jr.,W. L. Lam, et al., "Increased genetic damage in oral leukoplakia from high risk sites: potential impact on staging and clinical management," Cancer, 91(11), 2001, 2148-2155.

[17]. Sunali S Khanna and Freny R Karjodkar. Circulating Immune Complexes and trace elements (Copper, Iron and Selenium) as markers in oral precancer and cancer : a randomised, controlled clinical trial. Head \& Face Medicine, 2, 2006, 33

[18]. Renkonen J, Wolff H, Paavonen T. Expression of cyclo-oxygenase- 2 in human tongue carcinoma and its precursor lesions. Virchows Arch, 440, 2002, 594-597.

[19]. Banerjee AG, Gopalakrishnan VK, Bhattacharya I, Vishwanatha JK. Deregulated cyclooxygenase-2 expression in oral premalignant tissues. Mol Cancer Ther., 1, 2002, 1265-1271.

[20]. Atula T, Hedstrom J, Ristimaki A, Finne P, Leivo I, Markkanen- Leppanen M, et al. Cyclooxygenase-2 expression in squamous cell carcinoma of the oral cavity and pharynx: association to p53 and clinical outcome. Oncol Rep, 16, 2006, 485-490.

[21]. Neppelberg E, Johannessen AC. DNA content, cyclooxygenase- 2 expression and loss of E-cadherin expression do not predict risk of malignant transformation in oral lichen planus. Eur Arch Otorhinolaryngol, 264, 2007, 1223-1230.

[22]. Lysitsa S, Samson J, Gerber-Wicht C, Lang U, Lombardi T. COX-2 expression in oral lichen planus. Dermatology, 217, 2008, 150155 .

\section{Corresponding Author's Address:}

Dr N Rakesh, Reader

Dept. Oral Medicine, Diagnosis \& Radiology

M S Ramaiah Dental College \& Hospital

M S R I T Post, Bangalore - 560054

Email id: drnrakesh@ gmail.com, Contact no: 9448507494 